\title{
A SPECTRAL DECOMPOSITION THEOREM FOR CERTAIN HARMONIC ALGEBRAS
}

\author{
BY CHRISTOPHER I. BYRNES1
}

\author{
Communicated by I. Kaplansky, May 28, 1974
}

Introduction. Let $K$ be a simple ring with identity and let $A$ be a harmonic $K$-algebra with identity, where neither $K$ nor $A$ is assumed to be commutative. If one denotes the set of maximal ideals in $A$ by $\operatorname{Max}(A)$, then $A$ is strongly semisimple iff $S(A)=\bigcap_{M \in \operatorname{Max}(A)} M=(0)$. We assume that $A$ is strongly semisimple and note that this implies that $A$ is Jacobson semisimple. One may equip $\operatorname{Max}(A)$ with the hull-kernel topology, and we denote this space by $\max (A)=(\operatorname{Max}(A), \tau)$. We index $\operatorname{Max}(A)$ by the points of $\max (A)$; viz., if $p \in \max (A)$ then $M_{p} \in \operatorname{Max}(A)$ is the ideal corresponding to $p$. Since $A$ is harmonic, the space $\max (A)$ is a locally compact Hausdorff space [8] and, as usual, since $A$ has an identity, $\max (A)$ is compact. Teleman [8] has also shown that there exists a plastic, semisimple sheaf of local algebras such that $A \cong H^{0}(\max (A), \mathrm{A})$, where $H^{0}(\max (A), \mathrm{A})$ is the $K$-algebra of global sections of $A$. More generally, if $B$ is a strongly semisimple harmonic ring, then $B$ is isomorphic to a subring of $H^{0}(\max (B), B)$. However, this representation is too general for the applications we have in mind, as the elements of $A$ may take values in different simple rings. In addition, one of the characteristic features of the representation of rings by sections is that the topology of the base space can be extremely unmanageable [4]. In this note, we state sufficient conditions under which we are able to extract from Teleman's representation a second representation of $A$ as a ring of $K$-valued functions on a homogeneous, locally compact Hausdorff base space. It is obvious that, under mild restrictions, these conditions are also necessary.

REsults. We denote the group of $K$-algebra automorphisms of $A$ by $\operatorname{Aut}_{K}(A)$.

AMS (MOS) subject classifications (1970). Primary 16A72, 18F20, 54C40.

Key words and phrases. Harmonic ring, sheaf of local algebras, homogeneous topological space.

1 The author's graduate study is currently supported by an NSF Traineeship. 
Definition 1. If $M \subset \operatorname{Max}(A)$ we say that $A$ is $M$-transitive, provided whenever $M_{p}, M_{q} \in M$ there exists $\phi \in \operatorname{Aut}_{K}(A)$ with $\left(M_{p}\right) \phi=M_{q}$.

Definition 2. $M_{p} \in \operatorname{Max}(A)$ will be called geometric, provided $A / M_{p}$ $\cong K$; the set of geometric ideals is denoted by $(S)(A)$.

Those ideals $M_{p} \in \operatorname{Max}(A)-(S)(A)$ will be called hypergeometric, and the set of these ideals is denoted by $\mathfrak{D}(A)$. We denote the space $(\mathscr{S}(A), \tau \mid \mathcal{S}(A))$ by $\Gamma(A)$ and its complement in $\max (A)$ by $\Delta(A)$. If $F \subset \max (A)$ then the set

$$
A_{0}(F)=\{f \in A \mid \operatorname{supp}(f) \subset F\}
$$

is an ideal in $A$ [8]. The ideal $A_{0}(S(A))$ will be denoted simply by $A_{0}$.

Definition 3. $X \subset \operatorname{Max}(A)$ is said to be algebraically dense if whenever $f \in A$ and $f \notin M_{p}$, for all $M_{p} \in X$, then $f$ is invertible in $A$.

Since $A$ is harmonic, we have

THEOREM 1. If $X$ is algebraically dense, then its corresponding point set is dense in $\max (A)$.

Definition 4. If $X \subset \operatorname{Max}(A)$, then $A$ is said to be $X$ semisimple iff $\bigcap_{M_{p} \in X} M_{p}=(0)$.

Let $J(A)$ denote the Jacobson radical of $A$; one has

Lemma 1. If $f \in A$ is such that $1-f g$ is a right unit for all $g \in A$, then $f \in J(A)$.

COROllary 1. If $X$ is algebraically dense then $A$ is $X$ semisimple.

We have defined a decomposition of $\operatorname{Max}(A)$

$$
\operatorname{Max}(A)=\mathfrak{S}(A) \cup \mathfrak{Q}(A) \text {. }
$$

Now by definition, $A_{0} \subset \bigcap_{M_{p} \in \mathscr{F}_{(}(A)} M_{p}$, and hence $\mathfrak{Q}(A) \subset h\left(A_{0}\right) ; h\left(A_{0}\right)$ being the hull or locus of the ideal $A_{0}$. We shall say that $A$ is $K$-compact if $\mathfrak{Q}(A)=h\left(A_{0}\right)$.

Definition 5. $A$ is geometrically homogeneous iff

(i) $A$ is $(S)(A)$-transitive;

(ii) $(S)(A)$ is algebraically dense;

(iii) $A_{0} \neq(0)$.

TheOREM 2 (The DECOMPOSITION THEOREM). If $A$ is geometrically homogeneous then $A$ is $K$-compact. 
As an ideal in a harmonic $K$-algebra, $A_{0}$ is a harmonic $K$-algebra.

Corollary 2. If $A$ is geometrically homogeneous then $\Gamma(A) \cong$ $\max \left(A_{0}\right)$ and, hence, is locally compact Hausdorff.

Corollary 3. The maximal spectrum of $A$ may be decomposed as follows

$$
\max (A)=\Gamma(A) \bullet \Delta(A),
$$

where $\Gamma(A)$ is a locally compact dense subspace and $\Delta(A)$ is its closed complement. In fact, $A_{0}$ is the smallest ideal in $A$ having $\Gamma(A)$ as its colocus.

By Corollary 1, we have a faithful representation of $A$ as a subdirect product $\iota: A \rightarrow \Pi_{p \in \Gamma(A)} A / M_{p}$. We denote the image of $A$ under $\iota$ by $\hat{A}$ and, similarly, the image of $f$ in $\hat{A}$ by $\hat{f}$. Now, this representation of $A$ yields the isomorphisms

$$
A \cong H^{0}(\Gamma(A), \mathrm{A}), \quad A_{0} \cong H_{0}^{0}(\Gamma(A), \mathrm{A}) .
$$

As in the introduction, we also have a representation $A_{0} \cong H_{0}^{0}\left(\Gamma(A), A_{0}\right)$.

Since $\Gamma(A)$ is locally compact, and since the sheaf $A_{0}$ is plastic, a sheaf theoretic argument will prove

THEOREM 3. If $A$ is geometrically homogeneous, then $A$ is isomorphic to $H^{0}\left(\Gamma(A), \mathrm{A}_{0}\right)$.

DEFINITION 6. An automorphism of $\Gamma(A)$ is a bijection of sets, $\Phi: \Gamma(A) \rightarrow \Gamma(A)$, satisfying the following property: $\hat{f} \in \hat{A}$ iff $\hat{f} \circ \Phi \in \hat{A}$.

The set of all such maps, denoted by $\operatorname{Aut}(\Gamma(A))$, is clearly a group. The action of $\operatorname{Aut}(\Gamma(A))$ on $\Gamma(A)$ is to be computed "on the left." We have a map $\eta: \operatorname{Aut}(\Gamma(A)) \rightarrow \operatorname{Aut}_{K}(A)$ defined as follows: if $\Phi \in \operatorname{Aut}(\Gamma(A))$ then $\phi=\eta(\Phi)$ is the correspondence $(f) \phi=f \circ \Phi$ induced by $\hat{f} \rightarrow \hat{f} \circ \Phi$. In fact, we are able to prove

Theorem 4. Aut $(\Gamma(A))$ is canonically isomorphic to $\operatorname{Aut}_{K}(A)$.

One may also show

Corollary 4. Aut $(\Gamma(A)) \subset \operatorname{Hom}(\Gamma(A))$, where $\operatorname{Hom}(\Gamma(A))$ is the group of homeomorphisms of $\Gamma(A)$.

THEOREM 5. If $A$ is geometrically homogeneous then $A$ is isomorphic to the algebra of global sections in a plastic, semisimple sheaf $A_{0}$ over a homogeneous, locally compact Hausdorff base space, $\Gamma(A)$. Moreover, $A$ may 
be realized as an algebra of $K$-valued functions on $\Gamma(A)$.

EXAmple. Let $K=\mathbf{R}$ and let $A$ be an algebra of continuous, realvalued functions on $X$, a homogeneous, locally compact Hausdorff space. Under mild conditions on $A$ it is known that $\max (A) \cong \beta X$ and, further, that the algebra $A_{0}$ determines $X[5],[7]$. One notes also that the condition of R-compactness reduces to that of realcompactness, introduced by Hewitt in [3]. In particular, if $X$ is a smooth manifold and $A=C^{\infty}(X)$, then Teleman has shown that $\max (A) \cong \beta X$ [9]. Moreover, Bkouche has shown that $X$ is realcompact relative to $C^{\infty}(X)$, i.e. $\Gamma\left(C^{\infty}(X)\right) \cong X[1]$. This result may also be obtained from Theorem 5 and the result announced by Shanks in [7], thus the techniques developed by the author enable one to determine the fixed ideals in $A$ in a completely algebraic fashion. Pursell has also given an algebraic characterization of fixed ideals in concrete function rings in [6] by a different technique which requires a priori knowledge of the underlying space. More generally, Theorem 5 enables one to consider, in an applicable way, certain abstract harmonic algebras as function algebras. In fact, the author uses this theorem to characterize algebras of smooth functions defined on arbitrary smooth manifolds in [2] .

REMARK. Definition 3, in the commutative setting, is due to Bkouche, who originally called such a subset "very dense" (op.cit.). In more recent work Bkouche has used the term " $t$-dense", probably in order to avoid confusion with the current terminology employed by Grothendieck and Dieudonné. Proofs and a more detailed discussion will appear elsewhere.

\section{REFERENCES}

1. R. Bkouche, Ideaux mous d'anneau commutatif, C. R. Acad. Sci. Paris 260 (1965), 6496-6498. MR 31 \#1268.

2. C. I. Byrnes, Closed algebras of smooth functions, Bull. Amer. Math. Soc. (submitted).

3. E. Hewitt, Rings of real-valued continuous functions. I, Trans. Amer. Math. Soc. 64 (1948), 45-99. MR 10, 126.

4. K. H. Hoffman, Representations of algebras by continuous sections, Bull. Amer. Math. Soc. 78 (1972), $291-373$.

5. L. E. Pursell, Algebraic structures associated to a smooth manifold, Thesis, Purdue University, Fort Wayne, Ind., 1952.

6. - An algebraic characterization of fixed ideals in certain function rings,

Pacific J. Math. 5 (1955), 963-969. MR 18, 714.

7. M. E. Shanks, Rings of functions on locally compact spaces, Bull. Amer. Math. Soc. 57 (1951), 295. Abstract \#365. 
8. S. Teleman, Theory of harmonic algebras with applications to von Neumann algebras and the cohomology of locally compact spaces, Lecture Notes in Math., vol. 248, Springer-Verlag, Berlin and New York, 1971.

9. - La représentation des anneaux tauberiens discrets par des faisceaux, Rev. Roumaine Math. Pures Appl. 14 (1969), 249-264. MR 40 \#737a.

DEPARTMENT OF MATHEMATICS AND STATISTICS, UNIVERSITY OF MASSACHUSETTS, AMHERST, MASSACHUSETTS 01002 